\title{
Design of Broadband CNFET LNA Based on Extracted I-V Closed-Form Equation
}

\author{
Alireza Saberkari, Member, IEEE, Omid Khorgami, Member, IEEE, Javad Bagheri, Student Member, IEEE, \\ Morgan Madec, Member, IEEE, Seyed Mohsen Hosseini-Golgoo, and Eduard Alarcón-Cot, Senior Member, IEEE
}

\begin{abstract}
A procedure of extracting a closed-form userfriendly I-V equation for short channel carbon nanotube fieldeffect transistors (CNFET) in the saturation region is presented by employing a relation between CNFET parameters meeting the experimental results. The methodology is based on the Stanford model and ballistic relation of one channel CNFET. In this regard, the ballistic relation is simplified to a closed-form I-V equation, and then, the parameters are estimated through the fitting algorithm by means of ICCAP and least square (LS) method, respectively, and the obtained equation is verified by the experimental results given in the literature. Additionally, an extended quantitative noise analysis is performed at the circuit level and the noise sources implemented in Verilog- $A$ are added to the Stanford CNFET HSPICE model. Subsequently, with the accordance to the extracted I-V equation, a CNFET-based inductor-less broadband common-gate low noise amplifier (LNA) is designed theoretically and its results are confirmed in HSPICE based on the Stanford CNFET model, indicating a proper matching between analysis and simulation. The proposed CNFET-based LNA provides very high frequency bandwidth and also lower noise figure in comparison with its contemporary CMOS-based LNA, without any passive spiral inductor.
\end{abstract}

Index Terms-Carbon nanotube (CNT), field-effect transistor (FET), short channel, closed-form I-V equation, Low noise amplifier (LNA), radio frequency (RF), noise figure (NF), nanoelectronics.

\section{INTRODUCTION}

$\mathrm{C}$ ARBON nanotubes (CNTs) have the same properties of conductors and semiconductors. They are utilized in the field-effect transistors (FETs) as an alternative to conventional channels to provide CNFET devices, being considered as promising devices that could be substituted for CMOS by continuing scaling trends in semiconductor technology in the future [1]. Despite the intensive researches on the CNFETs in digital circuits and applications [2]-[4], few works can be found on CNFET applications in analog and RF domain; some of them are limited to presenting the lumped model for

Manuscript received October 11, 2017. This work has been partially funded by INSF.

A. Saberkari, O. Khorgami, J. Bagheri, and S. M. Hosseini-Golgoo are with the Microelectronics Research Lab., Department of Electrical Engineering, University of Guilan, Rasht, Guilan, Iran (e-mail: a_saberkari@guilan.ac.ir).

M. Madec is with the ICube Lab., University of Strasbourg, France (email: morgan.madec@unistra.fr).

E. Alarcon is with the Department of Electronics Engineering, Technical University of Catalunya, BarcelonaTech, Spain (e-mail: eduard.alarcon@upc.edu).
CNFET structures as impedance matching components via metal contacts [5]; other studies have focused on the CNFET unity gain frequency, $f_{\mathrm{T}}$, as a RF property which is given by $\nu_{\mathrm{F}} /\left(2 \pi L_{g}\right)$ for the Fermi velocity of $v_{\mathrm{F}}$ and gate length of $L_{g}[6]$, and also have performed CNFET device level considerations [7]. Moreover, RF building blocks features such as noise, linearity, power, and bandwidth have been reviewed in [8], [9]. Since the mentioned works indicate that CNFET can be utilized in RF applications, a simplified user-friendly I-V equation according to CNFET parameters is required at the first step of analog/RF integrated circuit (IC) design. In particular, apart from our preliminary work [10], there exists no user-friendly $\mathrm{I}-\mathrm{V}$ equation for CNFET as clear as MOSFET. In [11], [12], optimum analog CNFET-based circuit design is suggested; however, their design methods are just focused on sweeping the CNFET geometric design parameters. On the other hand, from experimental point of view, there are some experiments obtained from the fabricated CNFETs with the channel length of $350 \mathrm{~nm}-1 \mu \mathrm{m}$ in RF domain, which are categorized as long channel devices, and some noise analysis are performed [5], [13]. However, considering short channel devices with minimum size channel length is desirable to achieve the inherent capability of CNFETs in terms of noise and bandwidth in comparison with MOSFETs [14]. In overall, introducing a closed-form userfriendly I-V equation for short channel CNFET devices by employing a relation between CNFET parameters meeting the experimental results could be a primary requirement to have more details for CNFET-based RF IC design. Stanford CNFET model [15]-[18] provides a precise explanation of the electrical behavior of the intrinsic and additional doped CNTs. It has strong physical aspects such as transcapacitance network for intrinsic and doped CNTs, carrier scattering, parasitic capacitance, and screen effect. Apart from the mentioned features, it has a high accuracy with respect to the experimental results obtained from the fabricated CNFETs [18]. As well, it is notable that in the Stanford CNFET model, the transistor is considered as a short channel device with the channel length in the range of $10-100 \mathrm{~nm}$ in which the transport is quasi-ballistic. Additionally, a qualitative noise analysis is performed in [19] on short channel CNFETs based on the Stanford CNFET model.

As it is mentioned earlier, CNFET devices can be utilized in RF applications like low noise amplifiers (LNA), which are one of the essential blocks in receiver architectures. Moreover, the trend towards broadband LNAs in modern and future 
communication systems is due to covering multi-band frequencies, simultaneously, and reducing the total power by eliminating the extra components. Nevertheless, the design of broadband LNAs has conflicts in several aspects. One challenge is to achieve a low noise figure (NF) less than $3 \mathrm{~dB}$ as well as maintaining impedance matching over several gigahertz bandwidth. Utilizing the CNFET technology in conjunction with RF circuit techniques makes it possible to meet suitable circumstances for designing broadband CNFETbased LNAs. In order that, a theoretical background like prior technologies is needed. I-V equation at this background aids designers to acquire predictable consideration about CNFET's behavior. This work aims to extract a closed-form I-V equation for short channel CNFETs through which the design of RF circuits like broadband CNFET-based LNA would be easier.

This paper is organized as follows. In section II, a closedform I-V equation is extracted for short channel CNFETs in the saturation region based on the Stanford model and ballistic relation of one channel CNFET. After that and in section III, a quantitative noise analysis is performed at the circuit level for a short channel CNFET based on the Stanford model. The theoretical design of a CNFET-based broadband LNA is discussed, and then, its performance is compared to the CMOS-based LNA in sections IV and V, respectively. It is then followed by conclusion in section VI.

\section{CLOSED-FORM I-V EQUATION OF THE UNCORRELATED CHANNEL CNFET IN THE SATURATION REgION}

\section{A. Basis of CNFETS}

A 3D view of CNFET is illustrated in Fig. 1 (a) in which its channels are formed by intrinsic semiconductors of carbon nanotubes. The geometric parameters $D, W, N$, and $S$ indicate the diameter of the CNT, the transistor gate width, number of channels, and distance between centers of two adjacent CNTs, respectively. The relevance between these parameters is given by (1) [12]. Moreover, the parameter $D$ has an inverse relation with the threshold voltage of CNTs, $V_{t h}$, which is considered as half of the band gap energy, $E_{g}$, and expressed as (2) [20].

$$
\begin{gathered}
W=(N-1) S+D \\
V_{t h}=\frac{E_{g}}{2 q}=\frac{0.42}{D(n m)}(\mathrm{V})
\end{gathered}
$$

where $q$ is the electron charge. If the chirality vector is assumed as $C_{h}=\left(n_{1}, n_{2}\right)$, the parameter $D$ can be expressed as below [20]:

$$
D=\left|C_{h}\right| / \pi=\left(a_{c} / \pi\right) \sqrt{n_{1}^{2}+n_{1} n_{2}+n_{2}^{2}}
$$

where $a_{c}$ is the graphene lattice constant.

Fig. 1 (b) shows the cross-sectional view of the CNFET. The surface potential of the intrinsic channel, $\varphi_{S}$, is obtained by voltage dividing between the quantum capacitance per channel per unit length, $\mathrm{C}_{q}$, given by (4), and the gate-channel electrostatic oxide capacitance per channel per unit length, $\mathrm{C}_{\mathrm{ox}}$, as (5) [20].

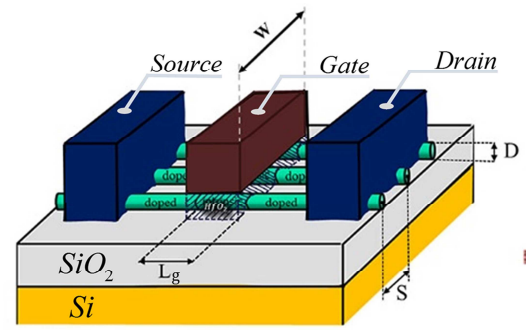

(a)

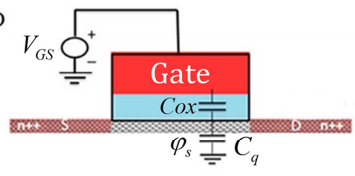

(b)

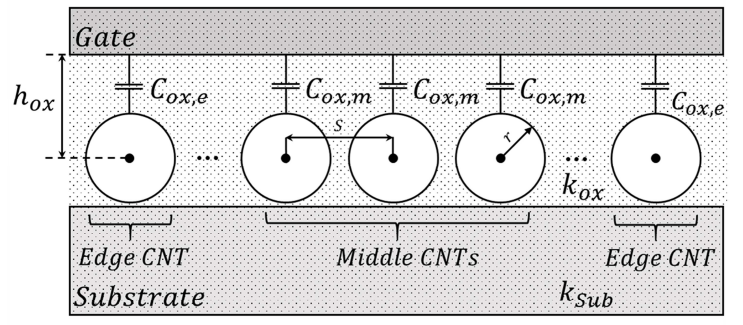

(c)
Fig. 1. CNFET: (a) 3D view, (b) cross-sectional view, and (c) front view of intrinsic CNTs.

$$
\begin{gathered}
C_{q}=\frac{2 q^{2}}{h v_{F}} \\
\varphi_{s}=\frac{C_{o x}}{C_{o x}+C_{q}} V_{G S}=t V_{G S}
\end{gathered}
$$

in which $t, v_{F}$, and $V_{G S}$ are the dividing factor, Fermi velocity of the CNT carriers, and its gate-source voltage, respectively. As a matter of fact, due to the position of the intrinsic CNTs, the oxide capacitors, i.e. gate-channel capacitors, are separated to middle, $C_{o x, m}$, and edge, $C_{o x, e}$, capacitors, as shown in the Fig. 1 (c) [16], [18]. The relation between these two oxide capacitors can be expressed as below:

$$
\begin{gathered}
C_{o x, m}=C_{o x, e}\left(1-\eta_{c}\right) \\
\eta_{c}=\frac{\ln \left(\frac{S^{2}+2\left(h_{o x}-r\right) \cdot\left[h_{o x}+\sqrt{h_{o x}{ }^{2}-r^{2}}\right]}{S^{2}+2\left(h_{o x}-r\right) \cdot\left[h_{o x}-\sqrt{h_{o x}{ }^{2}-r^{2}}\right]}\right)}{2\left[\cosh ^{-1}\left(\frac{2 h_{o x}}{D}\right)+\frac{k_{o x}-k_{S u b}}{k_{o x}+k_{S u b}} \ln \left(\frac{2 h_{o x}+2 D}{3 D}\right)\right]} \\
+\frac{k_{o x}-k_{S u b}}{k_{o x}+k_{S u b}} \ln \left(\frac{S^{2}+\left(h_{o x}+D\right)^{2}}{S^{2}+9 r^{2}}\right) \cdot \tanh \left(\frac{h_{o x}+r}{S-D}\right) \\
2\left[\cosh ^{-1}\left(\frac{2 h_{o x}}{D}\right)+\frac{k_{o x}-k_{S u b}}{k_{o x}+k_{S u b}} \ln \left(\frac{2 h_{o x}+2 D}{3 D}\right)\right]
\end{gathered}
$$

where $h_{o x}, k_{o x}, k_{S u b}$, and $r$ are the distance between the center of CNTs to the gate of the device, the gate oxide dielectric coefficient, the substrate dielectric coefficient, and the radius of the CNTs, respectively. The factor $\eta_{c}$ shows the electrostatic effect of the adjacent channels on the middle channel which makes its capacitor to be different from the edge capacitor. However, in accordance with [16] and by assuming $S$ greater than $20 \mathrm{~nm}, \eta_{c}$ is negligible. Therefore, the value of oxide capacitor per channel per unit length can be written as (8), in which $\varepsilon_{0}$ is vacuum permittivity. 


$$
C_{o x}=C_{o x, m}=C_{o x, e}=\frac{2 \pi k_{o x} \varepsilon_{0}}{\cosh ^{-1}\left(2 h_{o x} / \mathrm{D}\right)} \quad S>20 \mathrm{~nm}
$$

From electromagnetic point of view, the current of channels may be influenced by the adjacent channels because of the magnetic coupling between the near channels. It is feasible to specify these influences by calculating the magnetic field from nearest channel on target one, $B$, via the magnetic law, as (9).

$$
B=\frac{\mu_{0} I_{c} L_{c}}{2 \pi S \sqrt{S^{2}+L_{c}^{2}}}
$$

where $\mu_{0}$ and $L_{\mathrm{c}}$ are the permeability and half-length of each CNT, respectively. For a typical CNT, $I_{c}$ is the DC drainsource current of each channel in the range of micro ampere, $L_{c}$ and $S$ are in the range of nanometer, giving the acquired $B$ in the range of $10^{-4} \mathrm{~T}$. Such a small magnetic field cannot influence the current of the target channel [21].

In the light of the electrostatic and electromagnetic effects, generally, CNFET channels can be classified as correlated and uncorrelated channels. Namely, the current of the uncorrelated channel is not influenced by the adjacent channels currents. Indeed, for $S$ greater than $20 \mathrm{~nm}$ all channels have similar properties, i.e. $\eta_{c}$ is approximately equal to zero. By contrast, for the correlated channels, $S$ lower than $20 \mathrm{~nm}$, the current of target channel is affected by other channels via the electrostatic capacitances and electromagnetic fields.

\section{B. Description of the closed-form I-V equation}

As mentioned in prior sections, the Stanford model does not provide any straightforward closed-form I-V equation for the design of CNFET devices. For instance, in the Stanford model level 2, by considering the charge conservation equations [17], $I_{D}$ of the short channel CNFET with the drain-source voltage of $V_{D S}$ in the saturation region is expressed as below:

$$
\begin{gathered}
I_{D}=\frac{\sqrt{3} a_{C} \pi V_{\pi} C_{o x}\left(V_{G S}-\varphi_{S}\right)}{h} \sum_{k_{m}} \sum_{k_{l}} \frac{k_{l}}{\sqrt{k_{l}^{2}+k_{m}^{2}}} \\
\times\left[1+\frac{L_{g}}{\lambda_{o p}} \frac{\sqrt{\left(E\left(k_{m}, k_{l}\right)-\hbar \Omega\right)^{2}-E\left(k_{m}, 0\right)^{2}}}{E\left(k_{m}, k_{l}\right)-\hbar \Omega}\left(1+e^{-\frac{E\left(k_{m}, k_{l}\right)-q \varphi_{S}+q V_{D S}-h \Omega}{K T}}\right)\right]^{-1}
\end{gathered}
$$

where $V_{\pi}, \hbar \Omega, h, K T$, and $\lambda_{o p}$ are the carbon $\pi-\pi$ band energy, optical phonon-energy, Planck's constant, thermal energy, and optical phonon-scattering mean free path, respectively. The energy of states $E\left(k_{m}, k_{l}\right)$ is undeniable which is related to the wave number circumferential direction $k_{m}$ and wave number current flow direction $k_{l}$. In this case, $m$ and $l$ signify the $m$ th and $l$ th discrete sub-band in circumferential and current flow directions, respectively [17].

Nevertheless, to provide a closed-form I-V relation, considering some simplifications are required. The ballistic relation of one channel CNFET current is as follows [22]:

$$
I_{D}=\frac{4 q}{h} K T\left[\ln \left(1+e^{\left(q \varphi_{s}-E g / 2\right) / K T}\right)-\ln \left(1+e^{\left(q \varphi_{s}-q V_{D S}-\mathrm{Eg} / 2\right) / K T}\right)\right]
$$

For the saturation region, $V_{D S}>V_{G S}-V_{t h}$, it is irrefutable that the positive portion is dominated. Therefore, the saturation current can be expressed as below:

$$
I_{D}=\frac{4 q}{h} K T\left[\ln \left(1+e^{\left(q \varphi_{s}-E g / 2\right) / K T}\right)\right]
$$

Due to the value of $K T$, which is lower than $\left(q \varphi_{S}-E_{g} / 2\right)$, the term 1 in (10) is neglected in comparison to the exponential term. As a result, the I-V equation can be obtained by substituting (2), (4), and (5) in (12), as follows:

$$
I_{D} \approx 2 v_{F} \frac{C_{o x} C_{q}}{C_{o x}+C_{q}}\left(V_{G S}-V_{t h} / t\right)=g_{C}\left(V_{G S}-V_{t h} / t\right)
$$

where $g_{c}$ is the transconductance of each CNT. Therefore, the ideal I-V equation for the short channel CNFET with $N$ uncorrelated channels can be written as below:

$$
I_{\mathrm{D}} \approx N g_{C}\left(V_{\mathrm{GS}}-V_{t h} / t\right)
$$

\section{Validation of the closed-form I-V equation}

Since the Stanford model is available for ballistic short channel CNFETs, in order to validate the obtained closed-form equation given by (14), an optimization and curve fitting algorithm is applied on the CNFET by targeting the Stanford model. For doing it, the estimated closed-form I-V formula output is compared with the target system output, Stanford model, and the generated error is used to adjust the estimated formula coefficients.

The relation between the drain saturation current, $I$, and gate-source voltage, $\mathrm{v}$, of the transistor in the ballistic transport is as follows:

$$
I=\alpha\left(\mathrm{v}-\mathrm{v}_{t h}^{\prime}\right)^{p}
$$

in which $\mathrm{v}_{\text {th }}^{\prime}$ is the threshold voltage of CNFET. The exponent characteristic, $p$, must be between 1 and 1.5 [23]. There are three parameters to fit, $p, \alpha$, and $\mathrm{v}_{t h}^{\prime}$. The ICCAP software is used to fit these three parameters, simultaneously. First, the results provided by the Stanford model are imported to ICCAP as a measurement. Then a Verilog-A model involving Eq. (15) is implemented. ICCAP, coupled with Spectre performs simulations on the Verilog-A model, adjusts the parameters step by step in order to match the measurement. Fig. 2 illustrates the estimated parameter $p$ versus $N, D$, and $S$ which are swept in practical ranges. As it is obvious, the abundance of $p$ reaches the maximum value where it is between 0.94 and 1. As a result, it is considered that $p$ is approximately equal to 1. Therefore, (15) can be updated as below:

$$
I=\alpha\left(\mathrm{v}-\mathrm{v}_{t h}^{\prime}\right)=\theta_{1} \mathrm{v}+\theta_{0}
$$

where $\mathrm{v}^{\prime}$ th and $\alpha$ are equal to $\theta_{l}$ and $-\theta_{0} / \theta_{l}$, respectively.

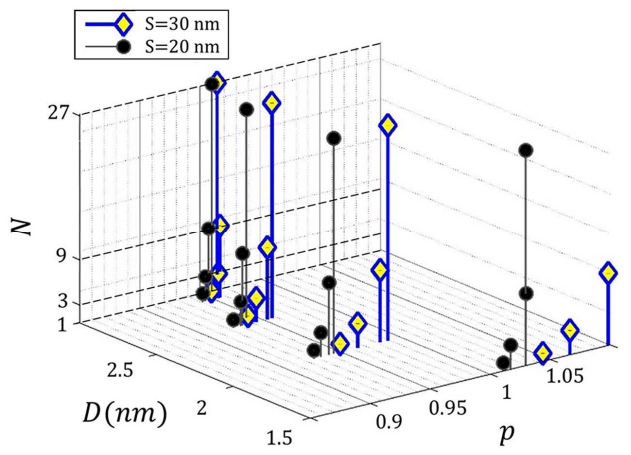

Fig. 2. Estimated parameter $p$ for different $N, D$, and $S$ 


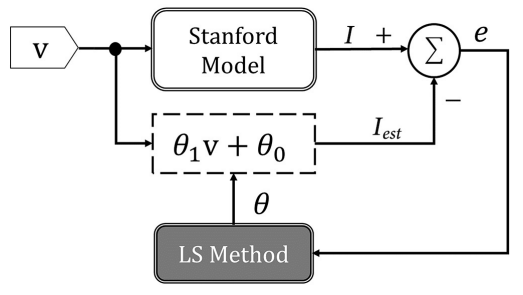

Fig. 3. Structure of the utilized system identifier.

As it can be seen, Eq. (16) is linear with respect to $\theta_{0}$ and $\theta_{1}$. Thus it can be identified by LS method. This structure is illustrated in Fig. 3, where the estimated current and error are denoted by $I_{e s t}$ and $e$, respectively. Estimated coefficients vector, $\boldsymbol{\theta}$, containing $\theta_{0}$ and $\theta_{1}$, is achieved by using the LS method as below:

$$
\boldsymbol{\theta}=V^{\dagger} I
$$

where $V$ represents the input matrix. Since the CNFET parameters are affected by geometric variations such as $D, S$, and $N$, changing one of the geometric parameters in each case for the input voltage from 0 to $2 \mathrm{~V}$, while considering the others fixed, and extracting the drain current, the estimated coefficients vector is obtained via the LS method, as given in Table I and Fig. 4. It is noted that the drain voltage of the CNFET is tuned at the saturation region. As it is shown in Fig. 5 , the estimated formula in (16) (dash line) has a good agreement with the target obtained from the Stanford model.

According to Fig. 5 (a), increasing $N$ causes an increase in the drain current. If the number of channels becomes $\beta$ times larger, the coefficient $\alpha$ will be increased by a factor of $\beta$, while $\mathrm{v}_{\text {th }}^{\prime}$ will be constant (Fig. 4 (a)). The reason can be attributed to the parallel channels. On the other hand, for the variations of $S$ greater than $20 \mathrm{~nm}$, the drain current will be constant (Fig. 5 (b)). In addition, the coefficient $\alpha$ and $\mathrm{v}_{\text {th }}^{\prime}$ will be constant, too.

In order to consider the effect of diameter on the drain current of CNFET, zigzag CNTs, i.e. CNTs with $n_{2}=0$ in (3), are considered. The increment of $n_{1}$ will cause to increase $D$ and $C_{o x}$ according to (3) and (8), respectively, and to decrease $V_{t h}$ based on (2), corresponding to the increment of coefficient $\alpha$ and reduction of $\mathrm{v}_{t h}^{\prime}$, obtained in Figs. 4 (b) and 5 (c).

From the obtained results, it can be concluded that the coefficient $\alpha$ (transconductance) is proportional to the multiplication of the number of channels and the capacitance. Moreover, according to the transconductance unit, it is noticed that $\alpha$ is approximately equal to $N C_{t} v_{C N T}$ and the saturated drain current given by (16) can be updated as below:

$$
I_{D}=N C_{t} v_{C N T}\left(V_{G S}-\mathrm{v}_{t h}^{\prime}\right) \quad V_{G S} \geq \mathrm{v}_{t h}^{\prime}
$$

in which, $v_{C N T}$ and $C_{t}$ are the carrier velocity and equivalent capacitance per unit length of the CNTs, respectively. If it is assumed that $C_{t}{ }^{-1}=C_{q}{ }^{-1}+\mathrm{C}_{\mathrm{ox}}{ }^{-1}$ and $\mathrm{v}_{t h}^{\prime}=\mathrm{V}_{\mathrm{th}} / t$, an acceptable matching will be found between the I-V closed-form equations given by (18) and (14) for uncorrelated short channel CNFETs. In order to validate the accuracy of the proposed closed-form I-V equation for CNFET in the saturation region, it is compared with the experimental results of [24], as it is shown in Fig. 6, in which the obtained mean-square error is in the order of $10^{-14} \mathrm{~A}$.
TABLE I

ESTIMATED COEFFICIENT

\begin{tabular}{cccccc}
\hline \hline Case No. & $N$ & $S(n m)$ & $D(n m)$ & $\alpha(A / V)$ & $v_{t h}^{\prime}(V)$ \\
\hline I. & 1 & 20 & 1.5 & $3.64 \mathrm{e}-5$ & 0.38 \\
N_variation & 3 & 20 & 1.5 & $1.08 \mathrm{e}-4$ & 0.38 \\
\hline II. & 3 & 20 & 1.5 & $1.08 \mathrm{e}-4$ & 0.38 \\
S_variation & 3 & 30 & 1.5 & $1.08 \mathrm{e}-4$ & 0.38 \\
\hline III. & 1 & 20 & 2.8 & $5.37 \mathrm{e}-5$ & 0.26 \\
D_variation & 1 & 20 & 1.5 & $3.64 \mathrm{e}-5$ & 0.38 \\
\hline \hline
\end{tabular}
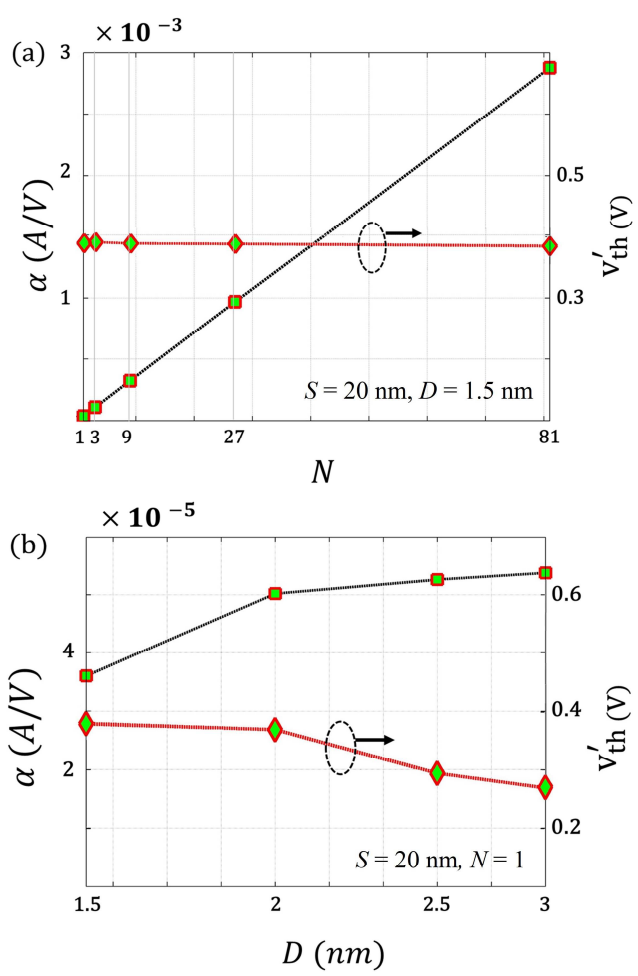

Fig. 4 Estimated coefficients $\alpha$ and $\mathrm{v}_{t h}^{\prime}$ for different, (a) $N$ and (b) $D$.

\section{OVERVIEW OF SHORT ChANNEL CNFET NoISE ANALYSIS}

A qualitative noise analysis on a one channel CNFET based on the Stanford CNFET model is performed in [19]. However, one channel CNFET cannot afford the RF application requirements like current and power demands. Hence, it is needed that a multiple channel CNFET is considered from noise analysis point of view to satisfy these necessities. This section provides a noise analysis for the short channel CNFET with $N$ channels based on the Stanford model by taking into account both the uncorrelated and correlated channels.

\section{Uncorrelated Channels}

As it is mentioned before, the CNFET channels are uncorrelated for $S$ greater than $20 \mathrm{~nm}$. In addition, two regions of saturation and triode are considered here.

\section{A. Saturation Region}

The saturation current in CNFETs is due to the invariance of carrier density while it appears in MOSFETs due to the pinch-off effect. Additionally, the short channel effect limiting the MOSFETs output resistance has no significant impact on the CNFETs. The simplified equivalent circuit of the CNFET 
based on the Stanford model is shown in Fig. 7 (a), where $R_{S}$ and $R_{d}$ represent the channel resistors at the source and drain regions, respectively, and $r_{o}$ is the output resistance of the device which is near to $100 \mathrm{M} \Omega$ per channel [18], [25]. It should be noted that the contact resistor of source/drain metal electrode described in [18] is smaller than $R_{S} / R_{d}$. Furthermore, since the magnetic inductance is about four times smaller than the kinetic inductance, the magnetic inductance is ignored in the CNFET Stanford model. As a result, the contact resistors and magnetic inductance are not taken into account in the equivalent circuit. According to [18], the CNFET intrinsic channel, CNFET_L1, in conjunction with the drain and source regions, CNFET_L2, forms a one channel CNFET. The equivalent transconductance of the CNFET_L2 can be expressed as follows:

$$
g_{C}=g_{\text {int }} /\left(1+g_{\text {int }} R_{S}\right)
$$

where $g_{c}$ and $g_{\text {int }}$ represent the CNFET_L2 and intrinsic transconductance, respectively. It is accepted that flicker and shot noises are the dominant noise sources in a short channel CNFET [19]. There are three main noise sources in the CNTFET_L2 consisting of the intrinsic channel equivalent noise (shot and flicker noises), and the doped channel drain/source region shot noises (Fig.7 (a)). However, the flicker noise is neglected at high frequencies because of its inverse relation with frequency. The equations of different noises per unit bandwidth can be expressed as below:

$$
\begin{gathered}
\overline{i_{n, \text { int }}^{2}}=2 q I_{D, L 2} F+\frac{\alpha_{H}}{n}\left(I_{D, L 2}^{2} / f\right) \\
\overline{i_{n, R_{S}}^{2}}=2 q I_{D, L 2} F_{d o p} \\
\overline{i_{n, R_{d}}^{2}}=2 q I_{D, L 2} F_{d o p}
\end{gathered}
$$

where $I_{D, L 2}, f, \alpha_{H}$, and $n$ are the drain current of CNFET_L2, frequency, technology-dependent constant, and number of charge carriers in the semiconductor, respectively. Moreover, $F_{d o p}$ and $F$ are doped and intrinsic channel's Fano factor, respectively. According to [19], $\alpha_{H}$ and $F_{d o p}$ are approximately equal to $10^{-4}$ and 0.3 by considering manufacturing results. On the other side, although there is no experimental results for intrinsic short channel shot noise, its theoritical model is widely reported in the litreture [26] in which $F$ is between 0 and 1 for the saturation current and has a reverse relation to $I_{D, L 2}$. By considering the high output impedance, the noise of $R_{d}$ has no significant effect on the CNFET_L2 equivalent current noise, $i_{n, c h t}$, shown in Fig. 7 (b). As a result, it can be expressed as below:

$$
\overline{i_{n, \text { cht }}^{2}}=G_{1} \overline{i_{n, R_{S}}^{2}}+G_{2} \overline{i_{n, \text { int }}^{2}}
$$

where $G_{1}$ and $G_{2}$ are the transfer gains which are equal to $\left(g_{\text {int }} R_{S} /\left(1+g_{\text {int }} R_{S}\right)\right)^{2}$ and $\left(1 /\left(1+g_{\text {int }} R_{S}\right)\right)^{2}$, respectively.

The total current of the CNFET with $N$ channels consists of the edge channels current, $I_{D, L 2, e}$, and middle channels current, $I_{D, L 2, m}$. Therefore, the equivalent noise includes two parts: edge channels noise, $i_{n, \text { cht,e, }}$ and middle channels noise, $i_{n, c h t, m}$, obtained by substituting edge Fano factor, $F_{e}$, edge intrinsic transconductance, $g_{i n t, e}$, and $I_{D, L 2, e}$ as well as middle Fano factor, $F_{m}$, middle intrinsic transconductance, $g_{i n t, m}$, and $I_{D, L 2, m}$
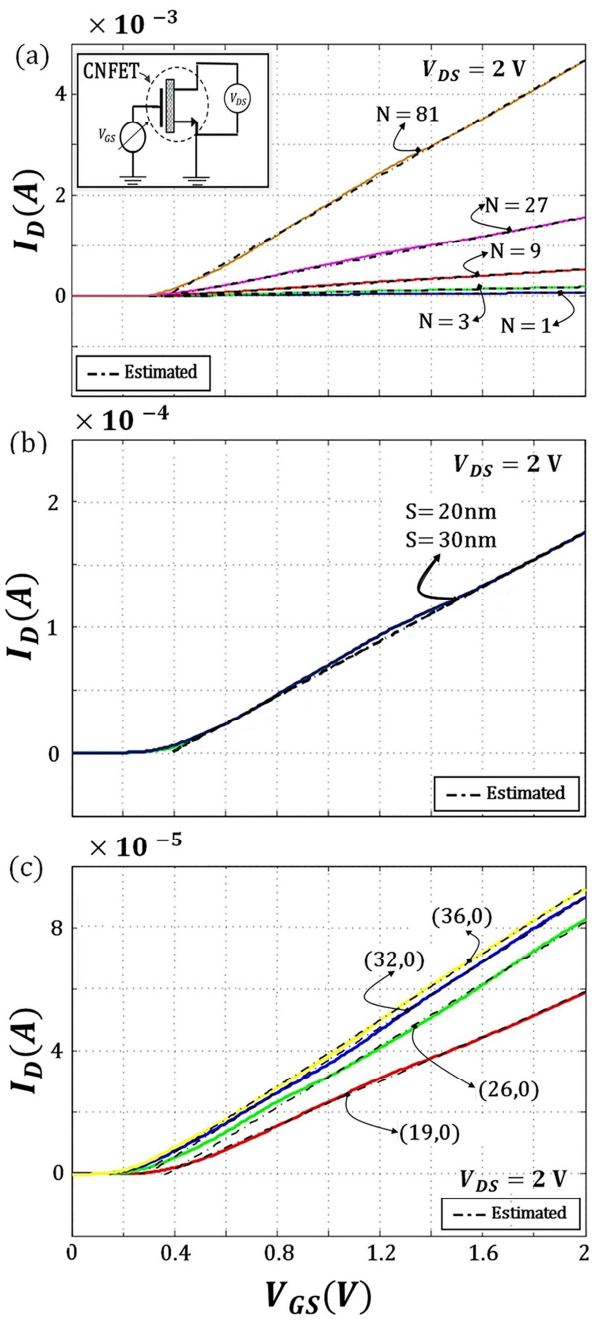

Fig. 5 Comparison between the estimated and Stanford model drain currents versus gate-source voltage of the CNFET for different (a) $N(S=20 \mathrm{~nm}, D=1.5$ nm), (b) $S(D=1.5 \mathrm{~nm}, N=3)$, (c) $D(S=20 \mathrm{~nm}, N=1)$.

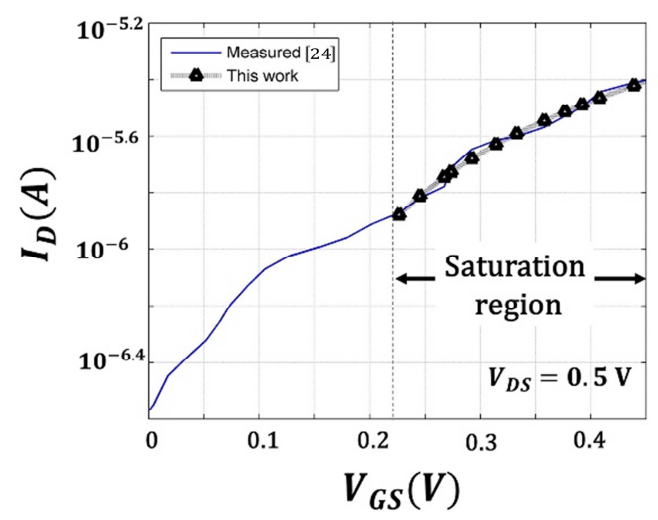

Fig. 6 Comparison between this work model and measurement results obtained from [24] ( $D=1.6 \mathrm{~nm}, N=1)$.

in Eqs. (20)-(23), respectively. As a result, total noise of the CNFET with $N$ channels, $i_{n, t o t}$, (Fig. 7 (c)), is equal to:

$$
\overline{i_{n, t o t}^{2}}=2 \overline{i_{n, c h t, e}^{2}}+(N-2) \overline{i_{n, c h t, m}^{2}}
$$

However, for uncorrelated channels, $i_{n, c h t, m}$ and $i_{n, c h t, e}$ will be equal [10]. Therefore, total noise of the CNFET with $N$ uncorrelated channels, $i_{n, t o t, u n}$, can be expressed as below: 


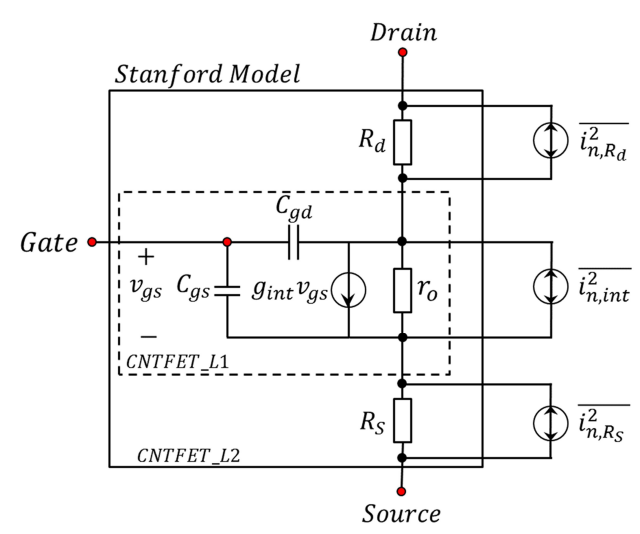

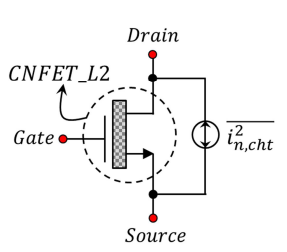

(b)

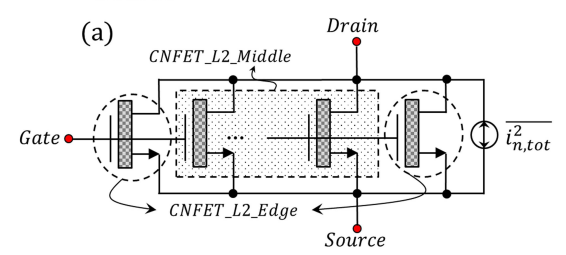

(c)
Fig. 7 (a) CNFET Small signal equivalent circuit and its internal noise current sources in the saturation region, (b) Equivalent compact noise source model considering the noise of CNFET_L2, (C) CNFET total noise sources with $N$ channels.

$$
\overline{i_{n, t o t, u n}^{2}}=N \overline{i_{n, c h t}^{2}}
$$

Substituting $I_{D, L 2}=I_{D} / N$, where $I_{D}$ is the current given by the closed-form I-V equation extracted in section II, and taking into account $q=K T / V_{T}$ ( $V_{T}$ is the thermal voltage), at high frequencies, (25) can be rewritten as below:

$$
\overline{i_{n, t o t, u n}^{2}}=4 K \operatorname{Kg}_{c} N H_{m}
$$

in which $g_{c}$ is equal to $C_{t} v_{C N T}$ obtained from (14) and (18). In addition, the coefficient $H_{m}$ is defined as below:

$$
H_{m}=\left(\frac{V_{G S}-\mathrm{v}_{\text {th }}^{\prime}}{2 V_{T}}\right)\left[\left(\frac{g_{\text {int }} R_{s}}{1+g_{\text {int }} R_{s}}\right)^{2} F_{\text {dop }}+\left(\frac{1}{1+g_{\text {int }} R_{s}}\right)^{2} F\right]
$$

It is clear that $H_{m}$ has a reverse relation to temperature, and hence, (26) is independent of temperature.

\section{B. Triode Region}

In the triode region, the intrinsic channel drain current has a linear behavior versus drain-source voltage. As a result, the intrinsic channel resistance $R_{d s}$ will be decreased with respect to $r_{o}$. The CNFET small signal model based on the Stanford model in the triode region is shown in Fig. 8 [18]. The equivalent current noise can be calculated as below:

$$
\begin{aligned}
\overline{i_{n, c h t}^{2}}= & {\left[\frac{R_{s}}{R_{d}+R_{d s}+R_{s}}\right]^{2} \overline{i_{n, R_{s}}^{2}}+\left[\frac{R_{d s}}{R_{d}+R_{d s}+R_{s}}\right]^{2} \overline{i_{n, \text { int }}^{2}} } \\
& +\left[\frac{R_{d}}{R_{d}+R_{d s}+R_{s}}\right]^{2} \overline{i_{n, R_{d}}^{2}}
\end{aligned}
$$

In addition, the total noise equation for $N$ uncorrelated channels is as follows:

$$
\overline{i_{n, \mathrm{cht}}^{2}}=\frac{N}{\left(R_{d}+R_{d s}+R_{s}\right)^{2}}\left(R_{s}^{2} \overline{i_{n, R_{s}}^{2}}+R_{d s}{ }^{2} \overline{i_{n, \mathrm{int}}^{2}}+R_{d}{ }^{2} \overline{i_{n, R_{d}}^{2}}\right)
$$

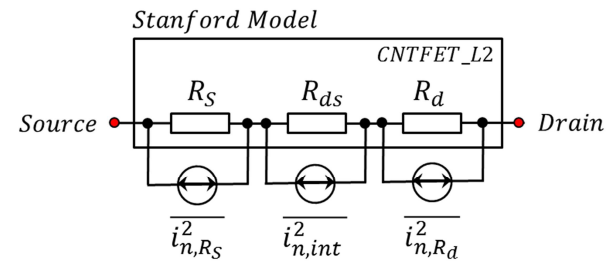

Fig. 8 CNFET Small-signal equivalent circuit and the internal noise current sources of the CNFET_L2 in the triode region.

If it is assumed that $R_{m}$ is the total resistance of $N$ uncorrelated channels of CNFET and by considering $R_{m, L 2}$, the CNFET_L2 equivalent resistance, equal to $R_{S}+R_{d s}+R_{d}\left(=N R_{m}\right)$, $I_{D, L 2}$ equal to $I_{D} / N\left(=V_{D S} / R_{m}\right)$, and $q=K T / V_{T}$, and neglecting the flicker noise at high frequencies, (29) can be simplified as (30), in which, $J_{m}$ is defined as (31).

$$
\begin{gathered}
\overline{i_{n, t o t, u n}^{2}}=4 K T \frac{1}{R_{m}} J_{m} \\
J_{m}=\frac{V_{D S}}{\left(2 V_{T}\right) R_{m, L 2}^{2}}\left[R_{s}^{2} F_{d o p}+R_{d s}{ }^{2} F+R_{d}{ }^{2} F_{d o p}\right]
\end{gathered}
$$

For a similar reason, (30) has no temperature dependency due to the inverse relation between $J_{m}$ and temperature.

\section{Correlated Channels}

The noise theoretical overview can be perused for correlated channels which their currents are influenced by neighboring channels current $\left(\eta_{c} \neq 0\right)$. In this circumstances, by taking (6), (7), and (10) into account, the relation among $I_{D, L 2, m}$ and $I_{D, L 2, e}$ can be expressed as below:

$$
I_{D, L 2, m}=I_{D, L 2, e}\left(1-\eta_{c}\right)
$$

Because of this difference between the edge and middle channels current, $F, G_{1}$ and $G_{2}$ will change, too. With regards to (24) and by considering $G_{1, e}, G_{2, e}$ and $G_{1, m}, G_{2, m}$ as the transfer gains of edge and middle channels, respectively, and $\sigma=F_{m}-F_{e}, \Delta G_{1}=G_{1, m}-G_{1, e}$, and $\Delta G_{2}=G_{2, m}-G_{2, e}$, total noise of the CNFET with $N$ correlated channels, $i_{n, t o t, C}$, is achieved as (33).

$$
\begin{aligned}
& \overline{i_{n, t o t, C}^{2}}=N \overline{i_{n, c h t, e}^{2}}+(N-2) \eta_{c} \overline{i_{n, c h t, e}^{2}} \\
& +2 q I_{D, L 2, e}(N-2)\left[\left(1-\eta_{c}\right) \sigma G_{2, e}+F_{d o p} \Delta G_{1}+F_{e} \Delta G_{2}+\sigma \Delta G_{2}\right]
\end{aligned}
$$

As it is clear in (33), the correlated term of $i_{n, t o t, C}$ corresponds to $\eta_{c}, \Delta G_{1}, \Delta G_{2}$, and $\sigma$. Hence, it is expected that (33) will become equal to (25) under uncorrelated situation when these factors proceed to zero.

\section{BROADBAND CNFET-BASED LNA DESIGN}

LNA architectures are generally categorized to commongate and common-source structures. The common-gate (CG) architecture demonstrates superior isolation and stability thanks to the lack of Miller effect of gate-drain capacitance, $C_{g d}$. In addition, due to its constant wideband input impedance, it can be considered as a good candidate for wide-band impedance matching, without using additional components, while preserving area consumption and avoiding from more resistance losses of on-chip inductors [27]. Beside the proper characteristics mentioned regarding the common-gate architecture, its noise performance is limited by the input 
matching condition due to fixing the device transconductance by the antenna output resistance. Therefore, noise reduction techniques are necessitated to be applied in order to improve the noise performance of CG LNAs for utilizing their wide bandwidth features [28]. There are some techniques to enhance the noise performance of the broadband LNAs (e.g., feedback and feedforward). The circuit noise is greatly dependent on the transconductance ratio of transistors (i.e., mismatch) in the feedforward techniques while this limitation is not significant in the feedback ones [29]. As a result, the negative feedback structure is used here to design the proposed broadband CNFET-based LNA.

The proposed broadband CNFET-based CG LNA topology is depicted in Fig. 9. It consists of two negative feedbacks which are applied by $M_{2}$ and $M_{3}$, while the signal is transferred towards the output by the common-gate transistor $M_{1}$. The common-source transistor $M_{2}$ boosts the transconductance of $M_{1} . R_{b}$ sets the extra path to the power supply to compensate the difference in the transconductance of $M_{2}$ and $M_{3}$. Since, the total capacitance of the input device in the CNFET technology is very low over the bandwidth there is no need to any inductor over the large bandwidth such as 3 to $38 \mathrm{GHz}$. As a result, the resistor $R_{p}$ is used here as the input terminal resistor instead of the conventional spiral inductors resulting in a broadband CNFET-based inductor-less CG LNA with reduced area. It is noticed that by carefully design of $R_{p}$, it is possible to reduce its noise effect. Moreover, transistor $M_{4}$, biased in triode region with equivalent resistance of $R_{L}$, provides an active load at the output terminal that used instead of conventional resistors to decrease the total noise. Finally, transistor $M_{5}$ buffers the output signal to provide a matching condition.

\section{A. Input Impedance Matching}

The in-band LNA impedance can be yield as (34):

$$
Z_{i n}=R_{p}\left\|\frac{1+g_{m 3} R_{b}\left(1+g_{m 1} R_{L}\right)}{g_{m 1}\left(1+g_{m 3} R_{b}+g_{m 2} R_{b}\right)}\right\| \frac{1}{S C_{i n}}
$$

in which, $C_{i n}$ is due to the parasite capacitors at the input which is very low. $g_{m i}$ denotes the total transconductance of transistor $M_{i}$ which is equal to $N g_{c}$ for $N$ uncorrelated channels. By assuming $g_{m 3} R_{b}\left(1+g_{m 1} R_{L}\right)$ and $g_{m 3}$ smaller than one and $g_{m 2}$, respectively, and considering $R_{p}$ at least 10 times than $R_{\text {Source }}$ (source resistance), (34) can be simplified as:

$$
Z_{i n}=\frac{1}{g_{m 1}\left(1+g_{m 2} R_{b}\right)}
$$

The input impedance $Z_{\text {in }}$ should be equal to $R_{\text {source }}$ for establishing the matching condition.

\section{B. Gain and Bandwidth}

As it is shown in Fig. 9, in terms of input impedance matching, the voltage gain from $V_{S}$ to $V_{\text {out }}$ can be calculated as follows:

$$
A_{V}=\frac{g_{m 1} R_{L}}{2}\left(\frac{g_{m 2}+g_{m 3}+1 / R_{b}}{g_{m 1} g_{m 3} R_{L}+g_{m 3}+1 / R_{b}}\right)
$$

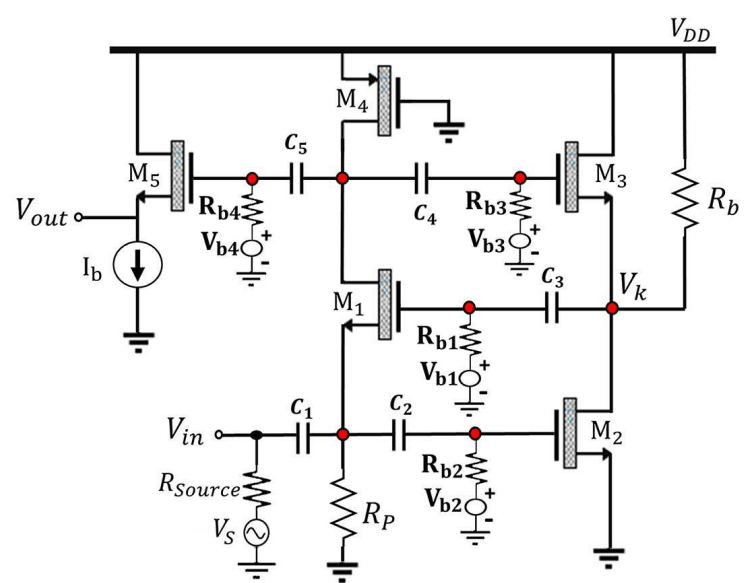

Fig. 9 Proposed broadband CNFET-based LNA topology.

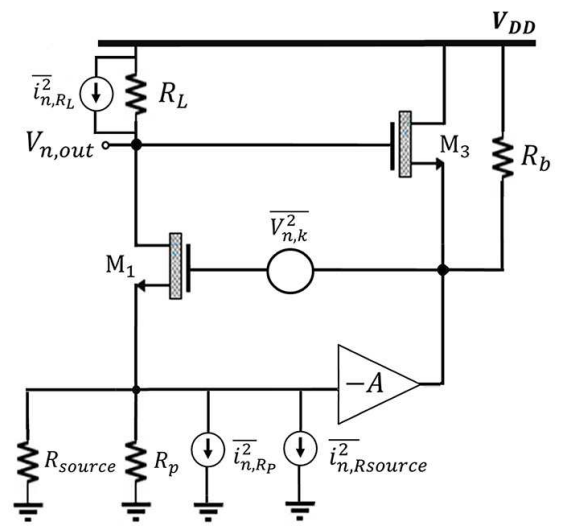

Fig. 10 Equivalent circuit with the noise sources.

According to the primary assumptions, (36) can be simplified as below:

$$
A_{V}=\frac{1}{2} g_{m 1} R_{L}\left(1+g_{m 2} R_{b}\right)=\frac{R_{L}}{2 R_{\text {Source }}}
$$

Moreover, the bandwidth is equal to $1 /\left(R_{L} C_{P}\right)$ in which $C_{P}$ is the parasite capacitance at the drain of $M_{1}$. We expect a high bandwidth due to the quantum capacitors of the CNFET [17].

\section{Noise Analysis}

The circuit noise sources consist of the CNFETs channel noise and the thermal noise of resistors. To calculate the noise figure, the ratio of the total noise due to all noise sources and the noise due to the source resistance is needed.

The voltage gain $V_{k} / V_{i n}$ is calculated as below:

$$
\frac{V_{k}}{V_{\text {in }}}=\frac{-g_{m 2}+g_{m 1} g_{m 3} R_{L}}{1 / R_{b}+g_{m 3}\left(1+g_{m 1} R_{L}\right)}=-g_{m 2} R_{b}
$$

Fig. 10 shows the equivalent circuit with its noise sources where the voltage gain $A$ equals $g_{m 2} R_{b}$ and the noises of $M_{1}$, $M_{2}, M_{3}$, and $R_{b}$ are defined as an accumulated noise source $V_{n, k}$ at node $k$. In addition, the voltage gain $V_{n, \text { out }} / V_{n, k}$ can be calculated as follows:

$$
\frac{V_{n, \text { out }}}{V_{n, k}}=\frac{-g_{m 1} R_{L}}{g_{m 1} R_{\text {source }}(1+A)+1}
$$

Due to the matching condition, $R_{\text {source }}=\left(g_{m 1}(1+A)\right)^{-1}$, (39) is simplified as below: 


$$
\frac{V_{n, \text { out }}}{V_{n, k}}=-g_{m 1} \frac{R_{L}}{2}
$$

The power spectral density of the noise components at the output of the first stage, the drain of $M_{1}$, within LNA's bandwidth can be obtained as follows:

$$
\begin{aligned}
& \left.\overline{V_{n, \text { out }}^{2}}\right|_{M 1}=\left(\frac{\left.\overline{i_{n, t o t, u n}^{2}}\right|_{M 1}}{g_{m 1}{ }^{2}}\right) \frac{\left(g_{m 1} R_{L}\right)^{2}}{4} \\
& \left.\overline{V_{n, \text { out }}^{2}}\right|_{M 2}=\left(\left.\overline{i_{n, t o t, \text {,un }}^{2}}\right|_{M 2} R_{b}^{2}\right) \frac{\left(g_{m 1} R_{L}\right)^{2}}{4} \\
& \left.\overline{V_{n, \text { out }}^{2}}\right|_{M 3}=\left(\left.\overline{i_{n, \text { tot }, \text { un }}^{2}}\right|_{M 3} R_{b}{ }^{2}\right) \frac{\left(g_{m 1} R_{L}\right)^{2}}{4} \\
& \left.\overline{V_{n, \text { out }}^{2}}\right|_{R_{b}}=\left(4 K T R_{b}\right) \frac{\left(g_{m 1} R_{L}\right)^{2}}{4} \\
& \left.\overline{V_{n, \text { out }}^{2}}\right|_{R_{L}}=4 K T J_{m 4} R_{L}
\end{aligned}
$$

The thermal current noises of $R_{p}$ and $R_{\text {source }}$ are transferred to the output with the same power gain as follows:

$$
\begin{aligned}
& \left.\overline{V_{n, \text { out }}^{2}}\right|_{R_{P}}=\left(4 K T \frac{1}{R_{p}}\right) \frac{R_{L}^{2}}{4} \\
& \left.\overline{V_{n, \text { out }}^{2}}\right|_{R_{\text {source }}}=\left(4 K T \frac{1}{R_{\text {source }}}\right) \frac{R_{L}^{2}}{4}
\end{aligned}
$$

Therefore, the noise figure is obtained as below:

$$
\begin{aligned}
N F & =1+\frac{\left.\overline{V_{n, \text { out }}^{2}}\right|_{M 1}+\left.\overline{V_{n, \text { out }}^{2}}\right|_{M 2}+\left.\overline{V_{n, \text { out }}^{2}}\right|_{M 3}}{\left.\overline{V_{n, \text { out }}^{2}}\right|_{R_{\text {source }}}} \\
& +\frac{\left.\overline{V_{n, \text { out }}}\right|_{R_{b}}+\left.\overline{V_{n, \text { out }}^{2}}\right|_{R_{L}}+\left.\overline{V_{n, \text { out }}^{2}}\right|_{R_{P}}}{\left.\overline{V_{n, \text { out }}^{2}}\right|_{R_{\text {source }}}} \\
& =1+\frac{H_{m 1}}{(1+A)}+\frac{A^{2} H_{m 2}}{g_{m 2} R_{\text {source }}(1+A)^{2}}+\frac{A^{2} H_{m 3}\left(g_{m 3} / g_{m 2}\right)}{g_{m 2} R_{\text {source }}(1+A)^{2}} \\
& +\frac{R_{b}}{R_{\text {source }}(1+A)^{2}}+\frac{4 J_{m 4} R_{\text {source }}}{R_{L}}+\frac{R_{\text {source }}}{R_{P}}
\end{aligned}
$$

The circuit has been designed in a way that $A$ or $g_{m 2} R_{b}$ are much higher than one, thus the noise effects of $M_{1}, M_{2}, M_{3}$, and $R_{b}$ on the noise figure would be insignificant. Moreover, the coefficients $H_{m 1}, H_{m 2}, H_{m 3}$, and $J_{m 4}$ are less than one, and hence, they can reduce the noise figure. All in all, the most important noise sources are $R_{p}$ and $R_{L}$ which should be designed, accurately.

According to (20), there is a dependency among the intrinsic shot noise and $F$. On the other hand, $N F$ is influenced by $F$. To determine this influence, the ratio of changes in $N F$ $(\triangle N F)$ to the variations of $F(\Delta F)$ due to the circuit components $M_{1}, M_{2}, M_{3}$, and $M_{4}$ are expressed as (49):

$$
\begin{gathered}
\left.\frac{\Delta N F}{\Delta F}\right|_{M_{1}}=\frac{V_{G S 1}-\mathrm{v}_{t h 1}^{\prime}}{2(1+A)\left(1+g_{\text {int } 1} R_{s 1}\right)^{2} V_{T}} \\
\left.\frac{\Delta N F}{\Delta F}\right|_{M_{2}}=\frac{A^{2}\left(V_{G S 2}-\mathrm{v}_{t h 2}^{\prime}\right)}{2 V_{T} g_{m 2} R_{\text {source }}(1+A)^{2}\left(1+g_{\text {int } 2} R_{s 2}\right)^{2}}
\end{gathered}
$$

$$
\begin{gathered}
\left.\frac{\Delta N F}{\Delta F}\right|_{M_{3}}=\frac{A^{2}\left(g_{m 3} / g_{m 2}\right)\left(V_{G S 3}-\mathrm{v}_{\text {th } 3}^{\prime}\right)}{2 V_{T} g_{m 2} R_{\text {source }}(1+A)^{2}\left(1+g_{\text {int } 3} R_{s 3}\right)^{2}} \\
\left.\frac{\Delta N F}{\Delta F}\right|_{M_{4}}=\frac{4 V_{D S 4} R_{d s 4}{ }^{2} R_{\text {source }}}{2 V_{T} R_{L}\left(R_{m 4, L 2}\right)^{2}}
\end{gathered}
$$

It is mentioned that, $\triangle N F$ is impressed a bit by $\Delta F$ in accord with the primary design assumptions expressed in this section.

\section{RESULTS}

The broadband CNFET-based LNA is designed and characterized in HSPICE based on the $32 \mathrm{~nm}$ Stanford model [15] and $1 \mathrm{~V}$ power supply. Although the Stanford model is validated by experimental results [18], it has not any noise sources. As a result, its compact noise model presented in [19] is extended here for $N$ channels CNFET and is taken into account via Veriloge-A for design of the LNA.

It is noted that, taking technological process into account for design parameters of CNFET devices is needed as practical aspects. Namely, although the CNT diameter variations and mispositioning of CNTs are considered as types of manufacturing process faults, the mechanism which is taken in [30] provides aligned arrays of CNTs perfectly while the diameters range from 0.5 to $3 \mathrm{~nm}$ within $10 \%$ standard deviations. In addition, although the diameter variations make unwanted metallic CNTs, they can be removed by thermocapillary resist as a promising approach [31]. Moreover, a majority of issues related to the fabrication process of CNFET has been solved and fabricated CNFETs are scaled down with minimum $S$ equal to $4 \mathrm{~nm}$ [32]. On the other hand, increasing the CNTs density in the form of high performance CNFETs for the future electronics looks promising. For instance, the CNTs density is enlarged up to 45-55 CNTs/ $\mu \mathrm{m}$ [33]. Due to these facts, it is significant to study the capability of systems designed in forthcoming technology. In this regard, the values of $D, S$, and CNTs density are set to $3 \mathrm{~nm}, 20 \mathrm{~nm}$, and $51 \mathrm{CNTs} / \mu \mathrm{m}$, respectively. The maximum transconductance per channel is reached by maximizing the practical diameter; hence, $D$ is set to $3 \mathrm{~nm}$. On the other hand, in order to have uncorrelated channels, $S$ is set to $20 \mathrm{~nm}$. As a result, the major design factor is the parameter $N$. In the design, we select the gate-source bias voltage; then the desired total current and transconductance can be obtained by selecting the proper $N$ based on the acquired closed-form I$\mathrm{V}$ equation. In this regard, the ratio of $g_{m 2} / g_{m 3}$ is almost equal to $N_{2} / N_{3}$ in which $N_{2}$ and $N_{3}$ are the number of channels of the transistors $M_{2}$ and $M_{3}$, respectively. It is noted that $N_{2}$ is set to be considerably much greater than $N_{3}$. Alternatively, we set $g_{m 3} R_{b}=0.01$ which is stemmed from $R_{b}=40 \Omega$ and $N_{3}=6$. These terms lead us to reach our primary assumptions, i.e. $g_{m 3} R_{b}(1+$ $\left.g_{m 1} R_{L}\right)<<1$ and $g_{m 3}<<g_{m 2}$. The design parameters and Stanford model technology configuration are listed in Tables II and III, respectively.

Fig. 11 illustrates $\triangle N F / \Delta F$ due to each component of the LNA. As it can be seen, small variation of $F$ will not cause significant variation in $\triangle N F$. For example, the variation of 0.1 for $F$ will cause the maximum changes about 0.3 for $\triangle N F / \Delta F$. Thus, the $\triangle N F$ will be equal to 0.03 which cannot have a significant influence on the total $N F$. 
TABLE II

DESIGN PARAMETERS OF THE BROADBAND CNFET-BASED LNA

\begin{tabular}{|c|c|c|c|c|c|c|c|c|c|}
\hline & \multicolumn{3}{|c|}{ CNFET Dimensions } & \multicolumn{2}{|c|}{ Bias Sources } & \multicolumn{2}{|c|}{ Operation Points (mA) } & \multirow{2}{*}{$\begin{array}{l}\text { Cap. } \\
\text { (pF) }\end{array}$} & \multirow[b]{2}{*}{$\begin{array}{r}\text { Res. } \\
(\Omega)\end{array}$} \\
\hline & $\mathrm{N}$ & $\begin{array}{c}\mathrm{S} \\
(\mathrm{nm})\end{array}$ & $\begin{array}{c}\mathrm{D} \\
(\mathrm{nm})\end{array}$ & $\begin{array}{l}\text { Voltage } \\
\text { (V) }\end{array}$ & $\begin{array}{c}\text { Current } \\
(\mathrm{mA})\end{array}$ & Simulation & Analytical & & \\
\hline M1 & 100 & 20 & 3 & $V_{b 1}=0.35$ & $I_{b}=2$ & $I_{D I}=0.216$ & $I_{D I}=0.218$ & $C_{1}=5$ & $R_{P}=500$ \\
\hline M2 & 3100 & 20 & 3 & $V_{b 2}=0.3$ & & $I_{D 2}=14.4$ & $I_{D 2}=14.5$ & $C_{2}=5$ & $R_{L}=900$ \\
\hline M3 & 6 & 20 & 3 & $V_{b 3}=0.8$ & & $I_{D 3}=0.038$ & $I_{D 3}=0.036$ & $C_{3}=5$ & $R_{b}=40$ \\
\hline M4 & 23 & 20 & 3 & $V_{b 4}=0.3$ & & & & $C_{4}=5$ & \\
\hline M5 & 450 & 20 & 3 & & & & & $C_{5}=5$ & \\
\hline
\end{tabular}

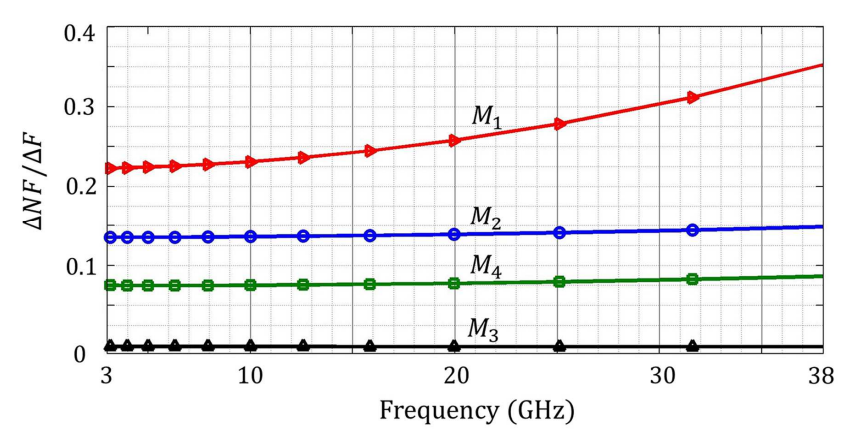

Fig. 11 The ratio of $\triangle N F$ to $\Delta F$ affected by each component of the LNA.
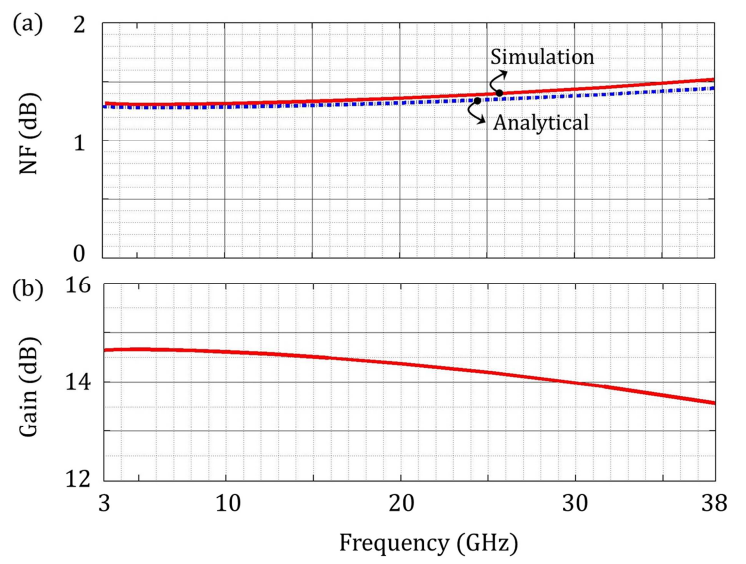

Fig. 12 Characterizations of the broadband CNFET-based LNA: (a) $N F$, and (b) Gain.

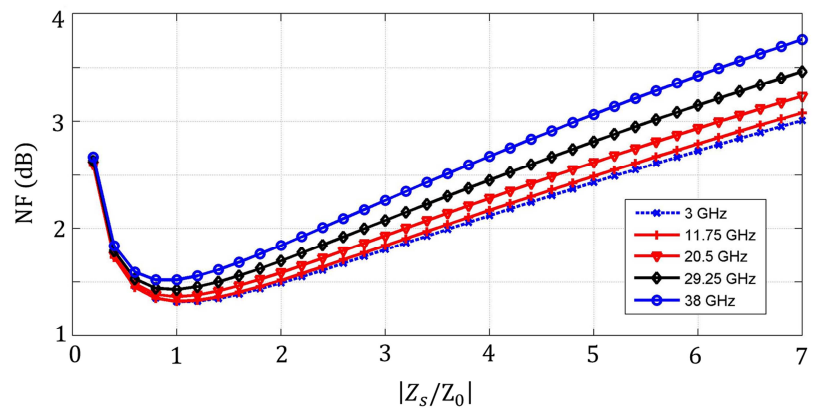

Fig. 13 Noise figure of the proposed LNA versus $\left|Z_{s} / Z_{0}\right|$.

The noise figure and gain of the LNA are shown in Fig. 12. Over the bandwidth ( 3 to $38 \mathrm{GHz}$ ); the noise figure is less than $1.5 \mathrm{~dB}$ and the gain equals $14.7 \mathrm{~dB}$ and drops just $1 \mathrm{~dB}$ over the entire bandwidth. In addition, there is an acceptable match between the analytical and simulation results. Fig. 13 shows the noise figure versus $\left|Z_{S} / Z_{0}\right|$ in which $Z_{s}$ is the source impedance and $Z_{0}$ is equal to $50 \Omega$. As it is obvious, the optim-
TABLE III

TeChnology CONFiguration OF THE Stanford Model

\begin{tabular}{cc}
\hline \hline Parameters & Value \\
\hline Chirality Vector & $(38,0)$ \\
Power Supply & $1 \mathrm{~V}$ \\
Gate Length & $32 \mathrm{~nm}$ \\
Dielectric Constant & 16 \\
Doped Source Side Length & $32 \mathrm{~nm}$ \\
Doped Drain Side Length & $32 \mathrm{~nm}$ \\
Gate Dielectric Thickness & $4 \mathrm{~nm}$ \\
Intrinsic CNT Mean Free Path & $200 \mathrm{~nm}$ \\
Doped CNT Mean Free Path & $12.5 \mathrm{~nm}$ \\
CNT Work Function & $4.5 \mathrm{eV}$ \\
Source/Drain Metal Contact Work Function & $4.6 \mathrm{eV}$ \\
\hline \hline
\end{tabular}

(a)

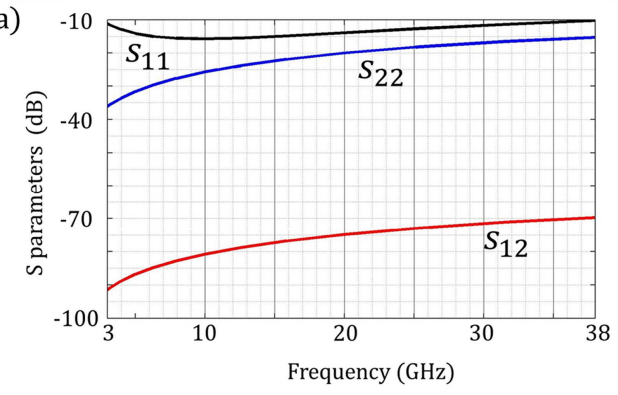

(b)

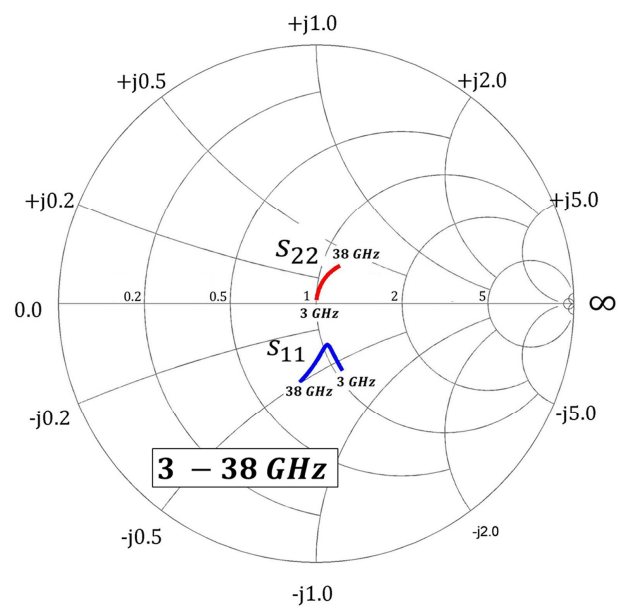

Fig. 14 Scattering parameters of the proposed broadband CNFET-based LNA: (a) $S_{11}, S_{22}$, and $S_{12}$ and (b) Smith chart.

um noise figure, $N F_{\min }$, is acquired around the source impedance of $50 \Omega$, approximately.

$S_{11}$ and $S_{22}$ are metrics of impedance matching with $50 \Omega$ resistor at the input and output of the LNA, respectively. To make impedance matching at the output, a buffer is used which has insignificant effect on the gain while establishing a better matching. As it is illustrated in Fig. 14 (a), the paramet- 
TABLE IV

PERFORMANCE OF THE BROADBAND CNFET-BASEd LNA IN COMPARISON WITH OTHER WORKS

\begin{tabular}{c|cccc}
\hline \hline Ref. & {$[28]$} & {$[29]$} & {$[34]$} & This work \\
\hline Technology & $180 \mathrm{~nm}$ & $180 \mathrm{~nm}$ & $450 \mathrm{~nm}$ & $32 \mathrm{~nm}$ \\
Data & CMOS & CMOS & CNFET & CNFET \\
Sim. & Exp. & Exp. & Sim. \\
Power & 1.8 & 1.8 & 2.5 & 1 \\
supply(V) & 15.3 & 12.6 & N.A. & 16 \\
P diss $_{\text {mW }}$ & 15.3 & -0.7 & -6 & 1.7 \\
IIP3(dBm) & -1.85 & $1.05-3.05$ & $1-1.2$ & $3-38$ \\
BW(GHz) & $3-6$ & $2.6-3.1$ & 8 & $0.4-1.3$ \\
NF(dB) & $3.5-3.6$ & 16.9 & 11 & $13.7-14.7$ \\
S21(dB) & $20.14-21$ & $<-10$ & $<-10$ & $<-10$ \\
S11(dB) & $<-11$ & N.A. & $<-18$ & $<-15$ \\
S22(dB) & $<-9$ & 4 & 5 & 0 \\
Number of & 6 & N.A. & N.A. & 0 \\
inductors & $18.75 \mathrm{nH}$ & N.A & \\
Total inductor & 18.78 & \\
\hline \hline
\end{tabular}
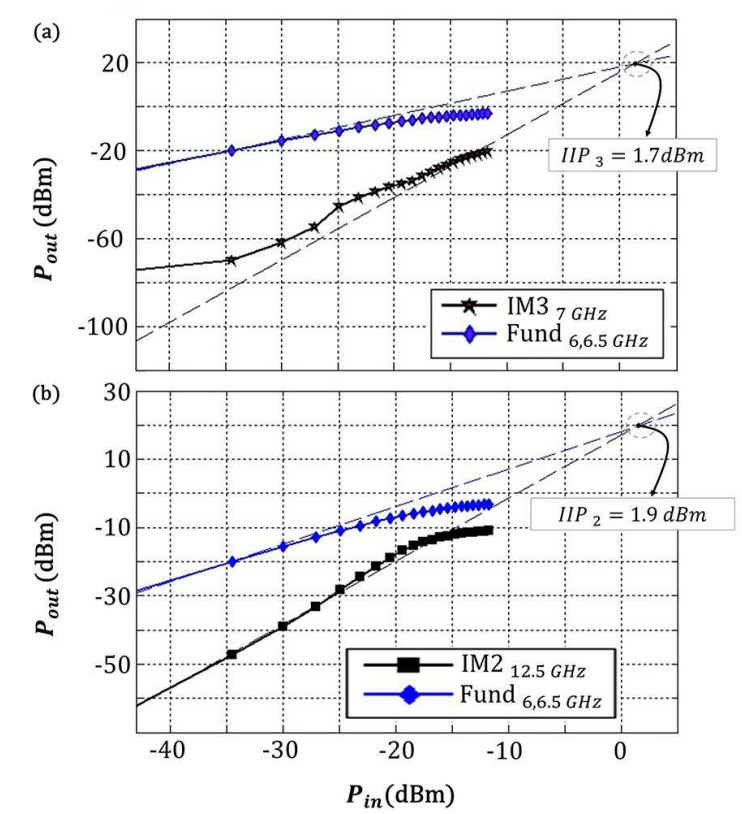

Fig. 15 Linearity characteristic of the broadband CNFET-based LNA: (a) $I I P_{3}$, and (b) $I I P_{2}$.

er at the entire frequency range, and hence, the system can be assumed as a unilateral device. As a result, the stability creteria can be obtained by checking $\left|S_{11}\right|$ and $\left|S_{22}\right|$. It is acceptable that in this circumstances, when $\left|S_{11}\right|$ and $\left|S_{22}\right|$ become less than 1 , the system is likely unconditionally stable. Additionally, for taking the phase behaviour into account, $S_{11}$ and $S_{22}$ are displayed on the Smith chart (Fig. 14 (b)). These graphs are approximately located around the center of Smith chart where the reflection coefficient is zero.

The linearity parameters $I I P_{3}$ and $I I P_{2}$ of the proposed CNFET-based LNA are shown in Fig. 15. For this purpose, two frequencies of $6 \mathrm{GHz}$ and $6.5 \mathrm{GHz}$ are applied as the input signals of the LNA. As it can be seen, $\mathrm{IIP}_{3}$ and $\mathrm{IIP}_{2}$ of 1.7 $\mathrm{dBm}$ and $1.9 \mathrm{dBm}$ have been reached.

In overall, Table IV lists the performance of the broadband CNFET-based LNA compared with recently published LNAs. The proposed circuit benefits from low $N F$, very high frequency range, and high linearity with moderate power consumption, while no passive spiral inductor has been utilized in its structure.

\section{CONCLUSION}

This paper has presented the extraction of a closed-form I-V equation for short channel CNFETs in the saturation region based on the Stanford model and ballistic relation of one channel CNFET. After simplifying the ballistic relation to a closed-form I-V equation, the coefficients have been estimated by ICCAP and LS method. Furthermore, an extended quantitative noise analysis has been performed at the circuit level and the noise sources implemented in Verilog-A are added to the Stanford model. Afterward, a CNFET-based inductor-less broadband CG LNA is designed theoretically based upon the extracted I-V equation and its results confirmed in HSPICE based on the Stanford CNFET model indicate a proper matchnig between analysis and simulation.

\section{REFERENCES}

[1] B. Sheu, P. C.-Y. Wu, and S. M. Sze, "Special issue on nanoele-ctronics and nanoscale processing," Proc. IEEE, vol. 91, no. 11, pp. 1747-1979, Nov. 2003.

[2] N. Patil et al., "Scalable carbon nanotube computational and storage circuits immune to metallic and mispositioned carbon nanotubes," IEEE Trans. Nanotechnol., vol. 10, no. 4, pp. 744-750, Jul. 2011.

[3] S. Lin, Y.-B. Kim, and F. Lombardi, "CNTFET-based design of ternary logic gates and arithmetic circuits," IEEE Trans. on Nanotechnol., vol. 10 , no. 2, pp. 217-225, 2011.

[4] G. Gielen et al., "Time-Based Sensor Interface Circuits in CMOS and Carbon Nanotube Technologies," IEEE Trans. on Circuits and Syst. I: Regular Paper, vol. 63, no. 5, pp. 577-586, Mar. 2016.

[5] M. Zhang, X. Huo, P. C. H. Chan, Q. Liang, and Z. K. Tang, "Radiofrequency transmission properties of carbon nanotubes in a field-effect transistor configuration," Electron Device Letters, vol. 27, no. 8, Aug. 2006.

[6] D.L. Pulfrey and L. Chen, "Examination of the high-frequency capability of carbon nanotube FETs," Solid-State Electron., vol. 52, no. 9, pp.1324-1328, Sept. 2008.

[7] M. Schroter et al., "A semiphysical large-signal compact carbon nanotube FET model for analog RF applications," IEEE Trans. Electron Devices, vol. 62, no. 1, pp. 52-60, Jan. 2015.

[8] M. Schroter, M. Claus, P. Sakalas, M. Haferlach, D. Wang, "Carbon nanotube FET technology for radio-frequency electronics: State-of-theart overview (invited)," IEEE J. Electron Devices Soc., vol. 1, no. 1, pp. 9-20, Jan. 2013.

[9] A. Alam et al., "RF linearity potential of carbon-nanotube transistors versus MOSFETs," IEEE Trans. Nanotechnol., vol. 12, no. 3, pp. 340351, May. 2013.

[10] O. Khorgami, A. Saberkari, J. Bagheri, S. Hosseini-Golgoo, and E. Alarcon-Cot, "Extracting a closed-form I-V equation and noise analysis for CNFET in analog/RF applications," in Proc. IEEE Int. New Circuits Syst. Conf. (NEWCAS'17), Jun. 2017, pp. 229 - 232.

[11] F. A. Usmani and M. Hasan, "Carbon nanotube field effect transistors for high-performance analog applications: an optimum design approach," Microelectronics J., vol. 41, no. 7, pp. 395-402, Apr. 2010.

[12] A. Imran, M. Hasan, A. Islam, and S. A. Abbasi, "Optimized design of a $32 \mathrm{~nm}$ CNFET based low power ultra-wide band CCII," IEEE Trans. Nanotechnology, vol. 11, no. 6, pp. 1100-1109, Nov. 2012.

[13] P. Sakalas et al., "High-frequency noise in manufacturable carbon nanotubetransistors," in Proc. IEEE Int. Conf. Noise and Fluctuations (ICNF'11), Jun. 2011, pp. 372-375.

[14] G.M. Landauer and J.L. Gonz'alez, "Carbon nanotube FET process variability and noise model for radiofrequency investigations," in 2012 12th IEEE Conf. Nanotech. (IEEE-NANO), Birmingham, UK, Aug. 2012, pp. 1-5.

[15] Stanford University CNFET model. [Online]. Available: http://nano.stanford.edu/model.php?id=23

[16] J. Deng and H. -S. P. Wong, "Modeling and analysis of planar-gate capacitance for 1-D FET with multiple cylindrical conducting channels," IEEE Trans. Electron Devices, vol. 54, no. 9, pp. 2377-2385, Sept. 2007. 
[17] J. Deng and H. -S. P. Wong, "A compact SPICE model for carbon nanotube field effect transistors including non-idealities and its application-Part I: model of the intrinsic channel region," IEEE Trans. Electron Devices, vol. 54, no. 12, pp. 3186-3194, Dec. 2007.

[18] J. Deng and H. -S. P. Wong, "A compact SPICE model for carbon nanotube field effect transistors including non-idealities and its application-Part II: full device model and circuit performance benchmarking," IEEE Trans. Electron Devices, vol. 54, no. 12, pp. 3195-3205, Dec. 2007

[19] G. M. Landauer and J. L. Gonzalez, "Radio-frequency performance of carbon nanotube-based devices and circuits considering noise and process variation," IEEE Trans. Nanotechnology, vol. 13, no. 2, pp. 228237, Mar. 2014.

[20] H. -S. P. Wong and D. Akinwande, Carbon nanotube and graphene device physics, Cambridge, Feb. 2011.

[21] J. P. Lu, "Novel magnetic properties of carbon nanotubes," Phys. Rev. Lett., vol. 74, no. 7, pp. 1123-1126, Feb. 1995.

[22] D. Akinwande, J. Liang, S. Chong, Y. Nishi, and H. -S. P. Wong, "Analytical ballistic theory of carbon nanotube transistors: experimental validation, device physics, parameter extraction, and performance projection," J. Appl. Phys., vol. 104, no. 12, pp. 124514, Dec. 2008.

[23] M. S. Lundstrom and Z. Ren, "Essential physics of carrier transport in nanoscale MOSFETs," IEEE Trans. Electron Devices, vol. 49, no. 1, pp. 131-141, Jan. 2002.

[24] A. Javey et al., "High-performance n-type carbon nanotube field-effect transistors with chemically doped contacts," Nano lett., vol. 5, no. 2, pp. 345-348, Jan. 2005.

[25] A. Bushmaker, M. R. Amer, and S. Cronin, "Electrical transport and channel length modulation in semiconducting carbon nanotube field effect transistors," IEEE Trans. Nanotechnol., vol. 13, no 2, pp.176 181, Mar. 2014.

[26] A. Betti, G. Fiori, and G. Iannaccone, "Shot noise suppression in quasione-dimensional field-effect transistors," IEEE Trans. Electron Devices, vol. 56, no. 9, pp. 2137-2143, Sept. 2009.

[27] A. Saberkari, S. Kazemi, V. Shirmohammadli, and M. Yagoub, "gmboosted flat gain UWB low noise amplifier with active inductor-based input matching network," Integration, the VLSI Journal, vol. 52, pp. 323-333, Jan. 2016.

[28] A. Saberkari, V. Shirmohammadli, and M. Yagoub, “A 3-6 GHz current reused noise canceling low noise amplifier for WLAN and WPAN applications," Wireless Personal Communications, vol. 86, no. 3, pp. 1359-1376, Feb. 2016.

[29] J. Kim, S. Hoyos, and J. Silva-Martinez, "Wideband common-gate CMOS LNA employing dual negative feedback with simultaneous noise, gain, and bandwidth optimization," IEEE Trans. Microwave Theory Tech., vol. 58, no. 9, pp. 2340-2351, Sept. 2010.

[30] N. Patil et al., "Wafer-scale growth and transfer of aligned single-walled carbon nanotubes," IEEE Trans. Nanotechnol., vol. 8, no. 4, pp. 498504, Jul. 2009.

[31] S. H. Jin et al., "Using nanoscale thermocapillary flows to create arrays of purely semiconducting single-walled carbon nanotubes," Nature Nanotech., vol. 8, no. 5, pp. 347-355, Apr. 2013.

[32] N. Patil, J. Deng, S. Mitra, and H. S. P. Wong, "Circuit-level performance benchmarking and scalability analysis of carbon nanotube transistor circuits," IEEE Trans. Nanotechnol., vol. 8, no. 1, pp. 37-45, Jan 2009.

[33] M. M. Shulaker et al., "Linear increases in carbon nanotube density through multiple transfer technique," Nano Lett., vol. 11, no. 5, pp. 1881-1886, Apr. 2011.

[34] M. Eron, S. Lin, D. Wang, M. Schroter, P. Kempf, “An L-band carbon nanotube transistor amplifier," Electron. Lett., vol. 47, no. 4, pp. 265266, Feb. 2011.

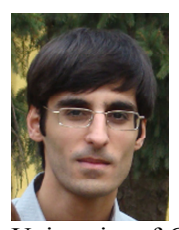

Alireza Saberkari received the M.Sc. and Ph.D. degrees both in electrical engineering from Iran University of Science and Technology (IUST), Tehran, Iran, in 2004 and 2010, respectively (all with honors). Since 2010, he has been with the department of electrical engineering at University of Guilan, where he became Associate Professor in 2015. He is the director of the microelectronics research lab. at the University of Guilan. During the period 2008-2009, he joined to the group of energy processing integrated circuits (EPIC), Technical University of Catalunya (UPC), Barcelona, Spain, as a visiting scholar. He has been involved in different national and international consisting Spanish and European Union R\&D projects. His fields of interest include the areas of analog, RF, and mixed-signal microelectronics with particular interest in onchip power management circuits, analog circuits for energy harvesting applications, linear and low-dropout regulators, wireless energy transfer, CMOS LNAs and RF power amplifiers.

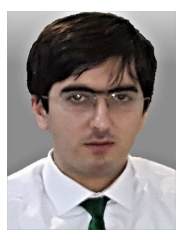

Omid Khorgami received the M.Sc. degree in electrical engineering-analog electronics from University of Guilan, in 2016. His research of interests include emerging nanoelectronic device \& mixed-signal design, identification procedure as $\mathrm{CAD}$ tool for design of integrated circuits, integrated RF building blocks circuit, low power \& low voltage CMOS integrated circuit design techniques, on-dhip power management circuits, circuit-level implementation of nonlinear controllers.

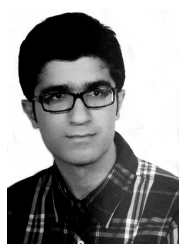

Javad Bagheri received the M.Sc. degree in electrical engineering-analog electronics from University of Guilan, in 2017. His research of interests include analog \& mixedsignal design, on-chip power management circuits especially analog \& digital low-dropout regulators, circuitlevel implementation of nonlinear controllers, identification procedure as CAD tool for design of integrated circuit, low power \& low voltage CMOS integrated circuit design techniques, RF CMOS LNAs and power amplifiers.

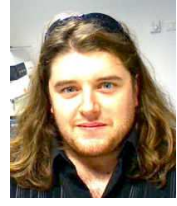

Morgan Madec received the Ph.D. degree in electronics engineering from the University of Strasbourg, France, in 2006. Since 2006, he has been with the same university (Télécom Physique Strasbourg) where he became Associate Professor in 2008. He is one of the members of the heterogeneous systems and microsystems (SMH) research team, ICube laboratory at the University of Strasbourg.

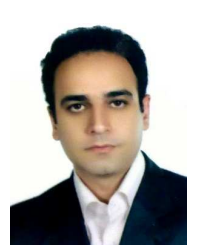

Seyed Mohsen Hosseini-Golgoo received the M.Sc. and Ph.D. degrees in electronic engineering from K. N. Toosi University of Technology, Tehran, Iran, in 2003 and 2009, respectively. $\mathrm{He}$ is currently an assistant professor of electronic engineering at University of Guilan, Rasht, Iran. He has founded a hi-tech company active in the field of ENose and gas sensor materials and technology. His research interests include machine olfaction and E-Nose, gas sensor fabrication and modeling, artificial neural networks and system identification, high temperature systems design and implementation.

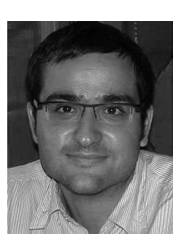

Eduard Alarcon received the M.Sc. (national award) and $\mathrm{Ph} . \mathrm{D}$. degrees (honors) in electrical engineering from the Technical University of Catalunya (UPC BarcelonaTech), Barcelona, Spain, in 1995 and 2000, respectively. He became Associate Professor at UPC BarcelonaTech in 2001. He has been visiting professor at the University of Colorado, Boulder $(2003,2006,2009)$ and KTH Stockholm (2011). His current research interests include the areas of on-chip energy management circuits, energy harvesting and wireless energy transfer, and nanotechnology-enabled wireless communications. Prof. Alarcón was IEEE CAS society distinguished lecturer (2009), elected member of the IEEE CAS Board of Governors (2010-2013), past chair the IEEE CAS IEEE technical committee for power circuits, co-editor of 5 journal special issues, co-organizer of 5 conference special sessions, held various TPC positions for IEEE conferences, and participates as Associate Editor for the IEEE TCAS-I, TCAS-II, JETCAS, JOLPE, and nano communication networks. 